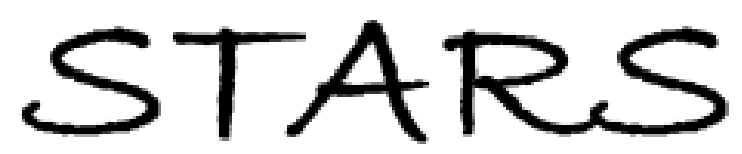

University of Central Florida

STARS

$1-1-2001$

\title{
Nanoscale p-n junction fabrication in silicon due to controlled dopant electromigration
}

\author{
Leonid Chernyak \\ University of Central Florida \\ Mikhail Klimov \\ University of Central Florida
}

Find similar works at: https://stars.library.ucf.edu/facultybib2000 University of Central Florida Libraries http://library.ucf.edu

This Article is brought to you for free and open access by the Faculty Bibliography at STARS. It has been accepted for inclusion in Faculty Bibliography 2000s by an authorized administrator of STARS. For more information, please contact STARS@ucf.edu.

\section{Recommended Citation}

Chernyak, Leonid and Klimov, Mikhail, "Nanoscale p-n junction fabrication in silicon due to controlled dopant electromigration" (2001). Faculty Bibliography 2000s. 2533.

https://stars.library.ucf.edu/facultybib2000/2533

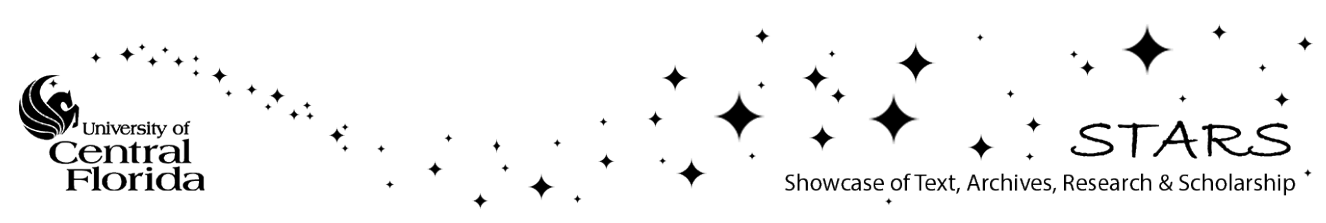




\section{Nanoscale $p-n$ junction fabrication in silicon due to controlled dopant electromigration}

Cite as: Appl. Phys. Lett. 78, 1613 (2001); https://doi.org/10.1063/1.1355009

Submitted: 04 December 2000 . Accepted: 17 January 2001. Published Online: 08 March 2001

Leonid Chernyak, and Mikhail Klimov

\section{ARTICLES YOU MAY BE INTERESTED IN}

A nanoscale pn junction in series with tunable Schottky barriers

Journal of Applied Physics 122, 134304 (2017); https://doi.org/10.1063/1.4994194

Laser ablation of bicomponent systems: A probe of molecular ejection mechanisms

Applied Physics Letters 78, 1631 (2001); https://doi.org/10.1063/1.1353816

Generalized Formula for the Electric Tunnel Effect between Similar Electrodes Separated by a Thin Insulating Film

Journal of Applied Physics 34, 1793 (1963); https://doi.org/10.1063/1.1702682

\section{Applied Physics Letters}

Mid-IR and THz frequency combs special collection 


\title{
Nanoscale $\boldsymbol{p}$ - $n$ junction fabrication in silicon due to controlled dopant electromigration
}

\author{
Leonid Chernyak ${ }^{\text {a) }}$ and Mikhail Klimov \\ Department of Physics, University of Central Florida, Orlando, Florida 32816-2385
}

(Received 4 December 2000; accepted for publication 17 January 2001)

\begin{abstract}
An external electric field (up to $10^{6} \mathrm{~V} / \mathrm{cm}$ ) was used for nanoscale $p$ - $n$ junction fabrication in $\mathrm{Si}$ doped with $\mathrm{Li}(\mathrm{Si}: \mathrm{Li})$ in situ in a scanning probe microscope. Creation of nano- $p$ - $n$ junctions was ascribed to the thermally assisted electromigration of $\mathrm{Li}^{+}$ions. Tunneling $I-V$ spectroscopy provided evidence for a conversion of the electrical conductivity type from $p$ to $n$. A local temperature increase during an electric field-induced $p-n$ junction fabrication was estimated to be up to $160{ }^{\circ} \mathrm{C}$. (C) 2001 American Institute of Physics. [DOI: 10.1063/1.1355009]
\end{abstract}

Since the appearance of semiconductor devices, the formation of $p-n$ junctions has been one of the most important processes in their fabrication. A $p-n$ junction is based on a nonequilibrium (thermodynamically unstable) distribution of dopants. Such distribution used to be obtained, in the early era of semiconductor device technology, by inducing dopant diffusion at high temperatures. Nowadays, this is done mostly by ion implantation. From the equilibrium thermodynamic point of view dopant distributions cannot be maintained without applying an external potential. In practice they are kinetically stabilized.

We have previously employed the phenomenon of controlled dopant electromigration for microscale bipolar device fabrication in semiconductor materials. Our approach was based on the finding that application of local, strong $\left(\sim 10^{6} \mathrm{~V} / \mathrm{cm}\right)$ electric fields, at ambient temperatures, to originally electrically homogeneous crystals of $\mathrm{Si}$ doped with $\mathrm{Li}(\mathrm{Si}: \mathrm{Li}),(\mathrm{Hg}, \mathrm{Cd}) \mathrm{Te}$, and $\mathrm{CuInSe}_{2}$ semiconductors induces nonequilibrium doping profiles in them (due to redistribution of impurities- $\mathrm{Li}^{+}$, or mobile native dopants- $\mathrm{Cu}^{+}$and $\mathrm{Hg}^{+}$), i.e., $\mu \mathrm{m}$-sized single and multiple diode (transistor) structures. ${ }^{1-5}$ Phototransistor action with amplification as well as electroluminescence were obtained, providing clear evidence for the presence of junctions, resulting from nonequilibrium doping profiles. The experiments on bipolar device fabrication, as described above, were carried out in situ in the scanning electron microscope (SEM) and the electron beam induced current (EBIC) technique was used to monitor the local changes in semiconductor electronic properties.

To scale down an electric field-induced $\mathrm{CuInSe}_{2}$-based device, the in situ experiments were carried out in ambient atomic force microscope ${ }^{6,7}$ using a conductive doped diamond tip. The minimum reported size for an electric fieldinduced $p-n$ junction was $\sim 60 \mathrm{~nm}{ }^{7}$

In this letter we report a nanoscale $p$ - $n$ junction fabrication in silicon using scanning probe microscopy (SPM). The $p-n$ junctions were created due to a thermally assisted electromigration of $\mathrm{Li}^{+}$ions. The obtained results provided an additional evidence that ion electromigration can be used to

${ }^{a)}$ Electronic mail: chernyak@physics.ucf.edu our benefit, as a relatively soft tool for nano-scale control over semiconductor electronic properties.

Samples of (111) Si with typical size $1 \times 1 \times 0.1 \mathrm{~cm}$ were cut from $p$-type floating zone wafers $(\rho=10 \Omega \mathrm{cm})$. Floating zone $\mathrm{Si}$ crystals have significantly higher $\mathrm{Li}$ ion mobility than Czochralski grown ones due to their lower oxygen content. The samples were covered by $30 \% \mathrm{Li}$ dispersion in mineral oil, dried in Ar atmosphere, and then annealed in vacuum $\left(10^{-5}\right.$ Torr $)$ at $400{ }^{\circ} \mathrm{C}$ for $3 \mathrm{~h}^{2}$ The annealing regime was chosen in such a way that highly resistive $\mathrm{Si}: \mathrm{Li}$ samples were obtained close to the surface. Still, the type of electrical conductivity did not change. After doping, the samples were polished using diamond pastes with a grain size down to $50 \mathrm{~nm}$.

Conductive tungsten tip of O-micron scanning probe microscope was used for in situ electric field-induced device fabrication. The experiments were carried out in air at room temperature. A single, up to $100 \mathrm{~ms} /$ duration negative voltage pulse, with a magnitude ranging from 30 to $50 \mathrm{~V}$, was applied to a SPM tip. Scanning tunneling microscopy (STM) mode of the scanning probe microscope was used instead of EBIC (see above) to monitor an electric field-induced fabrication of nano-device. This was done by scanning the region of interest before and after voltage application. In STM, it is sometimes difficult to distinguish between topographic and electrical signals, if the constant-current mode, based on the tunneling current feedback loop, is used. We have chosen, therefore, the tunneling current spectroscopy as a tool for $p-n$ junction detection. STM spectroscopy has been previously used for delineation of $p-n$ junctions fabricated in silicon wafers. $^{8}$

To apply an external voltage pulse in situ in SPM, the tunneling current feedback loop was disabled. This is because of current, ranging from several to $100 \mu \mathrm{A}$, which flows through the conductive tip/semiconductor system during an external pulse application. When the tunneling current feedback loop is disabled, and an external voltage pulse is applied, a tungsten tip is attracted to Si:Li surface, due to an electrostatic force. This force is sufficient for a physical contact, but does not lead to a visible tip or surface damage, as was confirmed by extensive high resolution $(\sim 3.5 \mathrm{~nm})$ scanning electron microscopy studies. We note that a physical contact between SPM W tip and Si surface is a necessary 


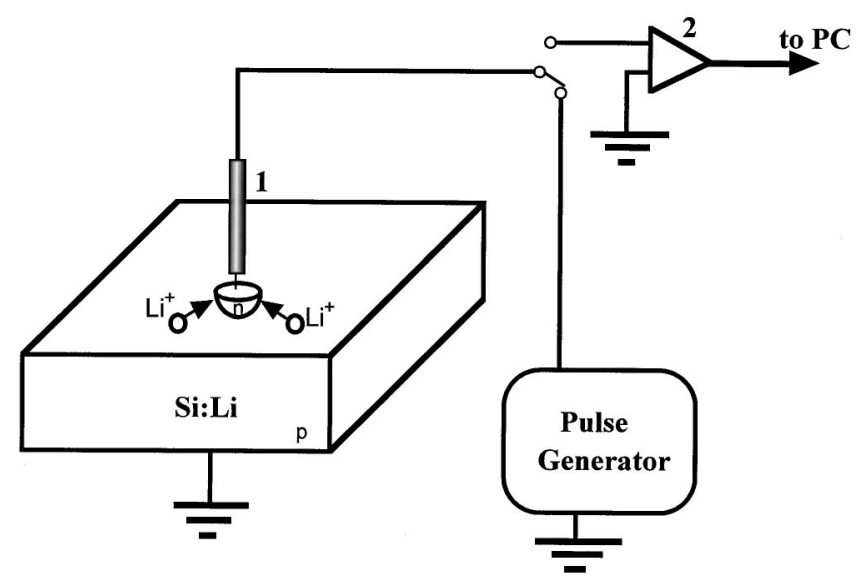

FIG. 1. Scheme of experimental setup: 1-SPM tungsten tip; 2-current amplifier. For an external voltage application by pulse generator, the SPM tunneling current feedback loop was disabled. This allowed up to $100 \mu \mathrm{A}$ current between W tip and Si:Li sample. Direction of electromigration for $\mathrm{Li}$ ions is shown by arrows. Region of $\mathrm{Li}^{+}$-ion collection is schematically shown as hemisphere. This region changes its conductivity type from $p$ to $n$, due to a shift of balance between boron-acceptor and Li-donor.

condition for structure creation, since it allows an applied electric field drop in the semiconductor, but not in the gap between the tip and the surface. The experimental setup, used in this research, is presented in Fig. 1.

Figure 2 presents the tunneling current contrasts from the region under investigation taken under $+2 \mathrm{~V}$, applied to the tip, before and after an external pulse application. The tunneling contrast in Fig. 2(a) indicates that the region of interest is originally electrically homogeneous. Figure 2(b) shows a tunneling contrast, created in the point of voltage application, after a negative 100-ms-long pulse with an amplitude of $\sim 50 \mathrm{~V}$ was applied. This contrast corresponds to a nano-scale $p$ - $n$ junction. For the $n$-type region, a higher positive than negative tip voltage has to be applied, to get tunneling current. The situation is opposite for the $p$-type region. Such an asymmetry is, indeed, seen in the tunneling $I-V$ curves in Fig. 3, measured in the $n$ - and $p$-type regions of the nano- $p-n$ junction, which is presented in Fig. 2. It must be noted that the threshold voltages at which a sharp increase of tunneling current is observed (cf. Fig. 3) are in agreement with the values calculated in Ref. 8 for the $n$ and $p$ regions.

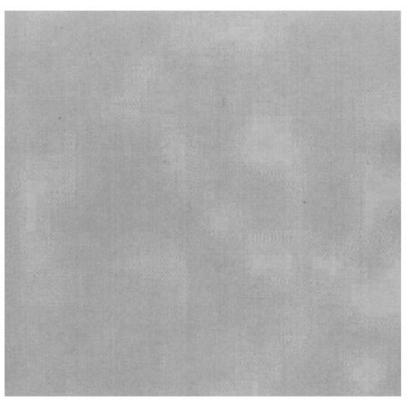

(a)

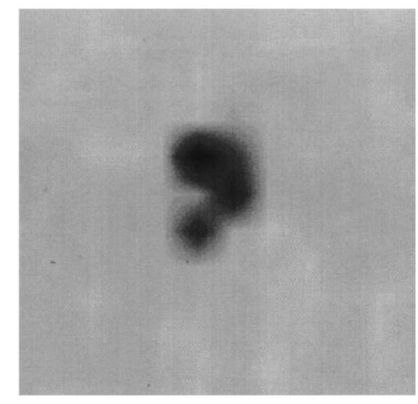

(b)
FIG. 2. Tunneling contrasts from the region of interest before (a) and after (b) 100-ms-long $-50 \mathrm{~V}$ pulse. A dark tunneling contrast, seen in (b) and taken under $+2 \mathrm{~V}$, applied to the SPM tip for tunneling current spectroscopy, corresponds to the $n$-type region. A bright background represents the $p$-type region, unaffected by an electric field [see also Fig. 2(a)].

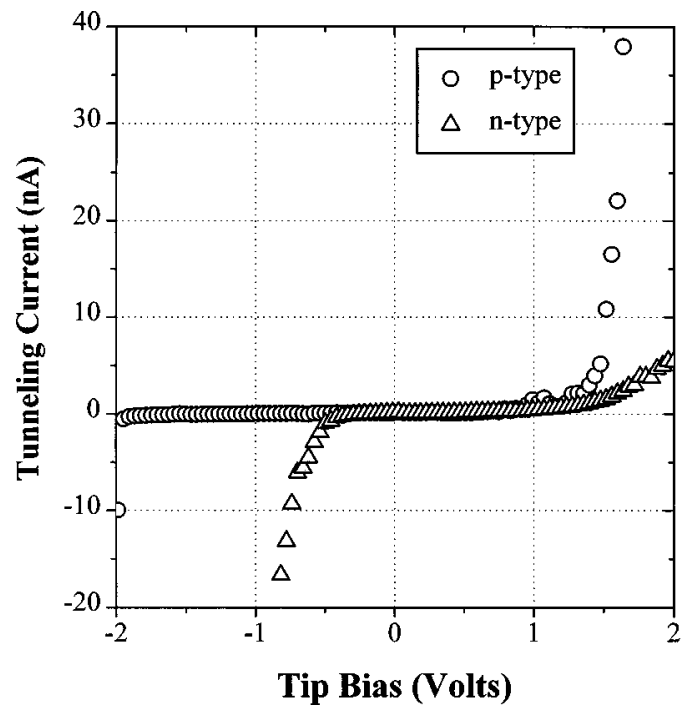

FIG. 3. Tunneling current-voltage curves from the $n$ - and $p$-type regions of the nano $p-n$ junction, presented in Fig. 2(b). For the $n$-type region, a higher positive than negative tip voltage has to be applied to get tunneling current. The situation is opposite for the $p$-type region.

We exclude a $\mathrm{Si}$ surface damage as a reason for the tunneling current contrast, observed in Fig. 2(b). This is because of the following.

(1) Much larger voltage application and power dissipation in $\mathrm{Si}$, which is not $\mathrm{Li}$ doped, does not lead to any tunneling contrast creation.

(2) The asymmetry of the $I-V$ curves in Fig. 3 testifies in favor of different electrical properties for the regions under investigation. Such an asymmetry cannot be obtained from the regions that are only topographically, but not electronically, different.

(3) High resolution SEM studies after an external voltage application revealed no visible surface damage.

Nano- $p$ - $n$ junction creation in $\mathrm{Si}: \mathrm{Li}$ is attributed to a redistribution of mobile $\mathrm{Li}^{+}$ions under the influence of an external electric field. Electric field application leads to a local temperature increase and consequent increase of mobility for $\mathrm{Li}^{+}$ions. Detailed description of ionic electromigration mechanism and its role in the electric field-induced device fabrication is given by us in Refs. $1-5 . \mathrm{Li}^{+}$ions are collected under the negatively biased STM tip due to their drift in an electric field (up to $10^{6} \mathrm{~V} / \mathrm{cm}$ ), induced at the W/Si interface. Although a polarity of a single voltage pulse, used in this work, corresponds to a forward bias of the W/Si Schottky barrier, our calculations show that an external voltage drop at the W/Si interface $(1-2 \mu \mathrm{m})$ is quite substantial $(\sim 90 \%)$, due to a strong compensation of boron with lithium, as was previously confirmed by secondary ion mass spectrometry (SIMS) measurements. ${ }^{2}$ Since before voltage application the sample under investigation was highly resistive $p$ type (background concentration of boron-acceptor was slightly higher than that of $\mathrm{Li}$-donor), accumulation of $\mathrm{Li}^{+}$ ion in the region adjacent to the $\mathrm{W}$ tip leads to a conversion of the electrical conductivity type there from $p$ to $n$ (cf. Fig. 1). The region abandoned by $\mathrm{Li}^{+}$ions is depleted with donors, and, therefore, its electrical conductivity should differ from the bulk, which remains unaffected by an applied elec- 
tric field. In practice, however, this does not happen, since in the spherical symmetry configuration, the Li-depleted region occupies much larger volume (it is proportional to the cube of the spherical radius - distance from the W tip; cf. Fig. 1) than the Li-rich one. Therefore, the shift of balance between the donors and acceptors in the Li-depleted region is insufficient to substantially affect the electrical conductivity.

The question of stability for an electric field-induced $p-n$ junction is of particular interest. As was mentioned in Refs. 1-4, the electric field-induced junctions are thermodynamically unstable, i.e., they remain in semiconductor material due to a low diffusion coefficient at room temperature. Based on the room temperature value of $\sim 10^{-15} \mathrm{~cm}^{2} / \mathrm{s}$ for $\mathrm{Li}^{+}$-ion diffusion coefficient in silicon, one may conclude that the electric field-induced nano- $p$ - $n$ junction should degrade within one month after an external voltage application. On the other hand, it is known that for some compound semiconductors the diffusion coefficient of native point defects $\left(\mathrm{Cu}^{+}\right.$ion in $\mathrm{CuInSe}_{2}$, for example) depends strongly on sample stoichiometry, ${ }^{9}$ which is, in turn, affected by an external electric field during a bipolar device fabrication in these materials. ${ }^{4}$ As a result, a degradation of an electric field-induced structure slows down. ${ }^{10}$ Additional experiments are required to understand the mechanism of nano- $p$ - $n$ junction annihilation.

In our previous work we have estimated the temperature, induced during microstructure creation, as a result of an external voltage application. ${ }^{11}$ This was done using different metal contacts of $\sim 50 \mu \mathrm{m}$ in diameter, to which an external voltage (and power), needed for structure fabrication, was applied. It must be noted, however, that because of nanoscale spherical contact, created between the tip and the top Si:Li surface, the above-referenced approach cannot be used here. Instead, we estimated the local temperature increase during nano- $p-n$-junction fabrication from the size of the electric field-induced structure.

From Fig. 2, the average distance, covered by $\mathrm{Li}^{+}$ions within $100 \mathrm{~ms}$ of an external voltage application, is $\sim 60 \mathrm{~nm}$ (spherical symmetry is assumed; cf. Figs. 1 and 2). From this distance, we find the velocity, $v$, of $\mathrm{Li}^{+}$ion to be $\sim 6$ $\times 10^{-4} \mathrm{~cm} / \mathrm{s}$. Remembering that $v=\mu E$ where $\mu$ = mobility of ion, and $E=$ strength of an applied electric field, and using $E=5 \times 10^{5} \mathrm{~V} / \mathrm{cm}$, we obtained $\mu \sim 1.2$ $\times 10^{-9} \mathrm{~cm}^{2} / \mathrm{V}$ s. Based on the experimental dependence of $\mathrm{Li}^{+}$-ion mobility on temperature, ${ }^{12}$ we find that overheating, created around the $\mathrm{W}$ tip as a result of an external pulse application is $\sim 160^{\circ} \mathrm{C}$. We note that this overheating is $\sim 2.5$ times smaller than that created in Si:Li during microdevice fabrication. ${ }^{1,2}$ The difference is explained by the fact that a much larger electric power (up to $3.75 \mathrm{~W}$ ) was dissipated in the latter case. ${ }^{2}$ Another important aspect to note is that the nano-scale point contact geometry does not play a significant role in sample overheating. This is because of the very large thermal diffusion length, $L$, in silicon. ${ }^{13}$ Under the experimental conditions described here, $L$ is $\sim 1200 \mu \mathrm{m}$. This means that the heat, created around the $\mathrm{W}$ tip during an external voltage application, is dissipated in the volume that is 15 orders of magnitude larger than that, defined by the probe/surface spherical symmetry.

The experiments on nanoscale- $p$ - $n$ junction fabrication were carried out on Si:Li samples in situ in a scanning probe microscope. A nano- $p-n$ junction was created as a result of the thermally assisted electromigration of $\mathrm{Li}^{+}$ions. Tunneling $I-V$ spectroscopy provided evidence for a conversion of the electrical conductivity type from $p$ to $n$. The local temperature increase during a $p-n$ junction creation was estimated to be up to $160^{\circ} \mathrm{C}$. To achieve control over device stability and find out practical applications, further understanding of the electric field-induced nanoscale tailoring of semiconductor electronic properties is required.

This research was supported in part by a UCF startup grant. L.C. thanks Dr. Vera Lyahovitskaya, Weizmann Institute of Science, for her help in Si doping.

${ }^{1}$ L. Chernyak, V. Lyakhovitskaya, and D. Cahen, Appl. Phys. Lett. 66, 709 (1995).

${ }^{2}$ L. Chernyak, V. Lyakhovitskaya, S. Richter, A. Jakubowicz, J. Manassen, S. Cohen, and D. Cahen, J. Appl. Phys. 80, 2749 (1996).

${ }^{3}$ L. Chernyak, A. Jakubowicz, and D. Cahen, Adv. Mater. 7, 45 (1995).

${ }^{4}$ L. Chernyak, K. Gartsman, D. Cahen, and O. M. Stafsudd, J. Phys. Chem. Solids 56, 1165 (1995).

${ }^{5}$ K. Gartsman, L. Chernyak, J. M. Gilet, R. Triboulet, and D. Cahen, Appl. Phys. Lett. 61, 2428 (1992).

${ }^{6}$ S. Richter, D. Cahen, S. R. Cohen, K. Gartsman, V. Lyakhovitskaya, and Y. Manassen, Appl. Phys. Lett. 73, 1868 (1998).

${ }^{7}$ S. Richter, Y. Manassen, and D. Cahen, Phys. Rev. B 59, 10877 (1999).

${ }^{8}$ R. Chapman, M. Kellam, S. Goodwin-Johansson, J. Russ, G. E. McGuire, and K. Kjoller, J. Vac. Sci. Technol. B 10, 502 (1991).

${ }^{9}$ G. Dagan, T. F. Ciszek, and D. Cahen, J. Phys. Chem. 96, 11009 (1992).

${ }^{10}$ D. Cahen and L. Chernyak, Adv. Mater. 9, 861 (1997).

${ }^{11}$ L. Chernyak, D. Cahen, S. Zhao, and D. Haneman, Appl. Phys. Lett. 65, 427 (1994).

${ }^{12}$ A. Zamouche, T. Heiser, and A. Mesli, Appl. Phys. Lett. 66, 631 (1995).

${ }^{13}$ L. Chernyak, Ph.D. thesis, Weizmann Institute of Science, Rehovot, Israel, 1995. 